\title{
COVID-19 pneumonia: a tailor-made dress for a Down syndrome patient
}

\author{
Simona Uggeri, ${ }^{1}$ Fabio Gilioli, ${ }^{1}$ Rossella Cosenza, ${ }^{1}$ Francesca Nasi, ${ }^{2}$ Stefano Moreali, ${ }^{1}$ Novella Guicciardi ${ }^{2}$ \\ ${ }^{1}$ Internal Medicine Department; and ${ }^{2}$ Radiodiagnostic Department, Mirandola Hospital, AUSL Modena, Italy
}

\begin{abstract}
We report here a case of coronavirus disease 2019 pneumonia in a 40-year-old Caucasian woman with Down syndrome admitted to the Internal Medicine Unit. She was initially treated with hydroxychloroquine and azithromycin. When respiratory conditions dramatically worsened, she was not admitted to the intensive care unit because of impaired cognitive function. Thus helmet-based continuous positive airway pressure was started. The respiratory conditions progressively improved, reaching spontaneous breathing.
\end{abstract}

\section{Case Report}

A 40-year-old Caucasian woman with Down syndrome (DS) was admitted to the Internal Medicine Unit, Hospital of Mirandola, last $26^{\text {th }}$ March. She was intellectually disabled, and she had a congenital solitary kidney with normal renal function. She had a body mass index of $35.8 \mathrm{~kg} / \mathrm{m}^{2}$. Other than these, previous medical history was unremarkable. She had a 4-day history of fever. She presented with fever $\left(38^{\circ} \mathrm{C}\right)$, dry cough, and pharyngodynia. Severe acute respiratory syndrome-related coronavirus 2 (SARS$\mathrm{CoV}-2$ ) positivity was confirmed with one nasal/throat

Correspondence: Simona Uggeri, Internal Medicine Department, Mirandola Hospital, via Fogazzaro 1, Modena, Italy. Tel.: +39.0535.602377.

E-mail: s.uggeri@ausl.mo.it

Key words: Severe acute respiratory syndrome-related coronavirus 2; continuous positive airway pressure; morphine; Down syndrome.

Conflict of interests: the authors declare no potential conflict of interests.

Ethics approval: not required.

Informed consent: the patient gave informed consent.

Received for publication: 27 September 2020.

Accepted for publication: 20 October 2020.

This work is licensed under a Creative Commons Attribution NonCommercial 4.0 License (CC BY-NC 4.0).

${ }^{\circ}$ Copyright: the Author(s), 2021

Licensee PAGEPress, Italy

Italian Journal of Medicine 2021; 15:59-63

doi:10.4081/itjm.2021.1377 swab test of SARS-CoV-2 by qualitative real-time reverse-transcriptase-polymerase-chain-reaction (RTPCR) assay. The initial physical examination revealed oxygen saturation $\left(\mathrm{SpO}_{2}\right)$ 95\% under ambient air, respiratory rate of 22 breaths/minute, blood pressure of $100 / 80 \mathrm{~mm} \mathrm{Hg}$, and pulse of $104 \mathrm{bpm}$. The laboratory findings showed elevated C-reactive protein $(3.7 \mathrm{mg} / \mathrm{dL}$, reference $<0.5)$ and low white blood cell count $\left(1800 \mathrm{~mL}^{-1}\right)$. On admission, a chest X-ray revealed multiple bilateral pulmonary infiltrates, in the upper and lower lobes, involved by consolidations (Figure 1). The comparison of laboratories findings during the hospitalization was presented in Table 1.

On day 1, she had dyspnea, hypoxemic acute respiratory failure $\left(\mathrm{paO}_{2} / \mathrm{FiO}_{2} 195\right)$, and oxygen supply through the Venturi mask, labeled at $28 \%$ fraction of inspired oxygen $\left(\mathrm{FiO}_{2}\right)$. Thus, hydroxychloroquine and azithromycin were started. She received hydroxychloroquine $400 \mathrm{mg}$ twice on day 1 , followed by $200 \mathrm{mg}$ twice per day on days $2-5$, and azithromycin $500 \mathrm{mg}$ once for 5 days. We have also administered low molecular weight heparin (40 mg once a day) for prophylaxis of deep vein thrombosis.

On day 3 , her body temperature returned to normal, but she still had dyspnea, and needed highflow oxygen $(8 \mathrm{~L} / \mathrm{min})$ through a Venturi mask. Thus, tocilizumab was given subcutaneously at $162 \mathrm{mg}$ administered in two simultaneous doses, one in each thigh (324 $\mathrm{mg}$ in total) because the endovenous formulation was unavailable.

On day 5 she presented uncontrollable respiratory distress $\left(\mathrm{paO}_{2} / \mathrm{FiO}_{2} 70\right)$ despite maximal oxygen supply. She was not admitted to the intensive care unit because of impaired cognitive function.

Thus, it was started a helmet-based continuous positive airway pressure (helmet CPAP) with positive 
end-expiratory pressure $10 \mathrm{~cm} \mathrm{H}_{2} \mathrm{O}$. Simultaneously we administered methylprednisolone $40 \mathrm{mg}$ /day for 7 days. She was given subcutaneously morphine $10 \mathrm{mg}$ per day for dyspnea and strong negative emotions, for five days, without any side effects. Carbon dioxide level increased on day 8 , with resolution in the following days after stopping using the helmet because helmet CPAP might facilitate $\mathrm{CO}_{2}$ rebreathing. On day 15 , the second chest-X-rays

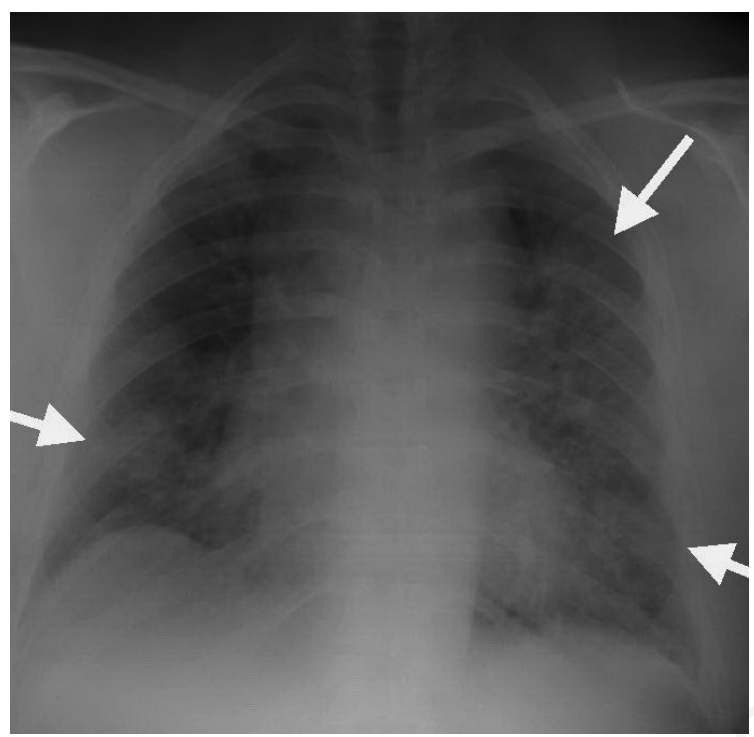

Figure 1. On admission to the hospital, the chest film shows ill-defined bilateral alveolar consolidation with peripheral distribution, in the right lower lobe and in the upper and lower left lobe (arrows).

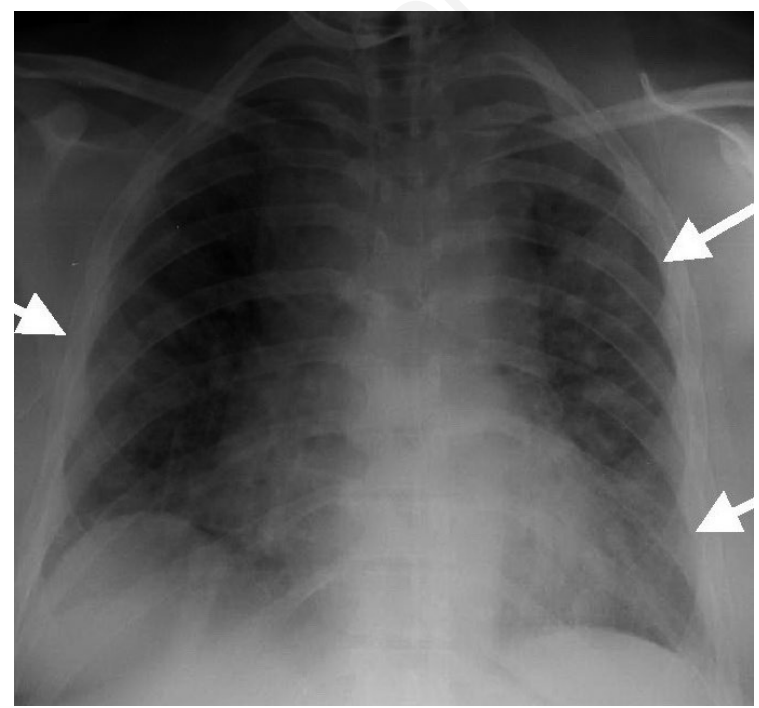

Figure 2. Chest X-ray with evidence of partial clearance (arrows) of bilateral pulmonary infiltrates was seen.

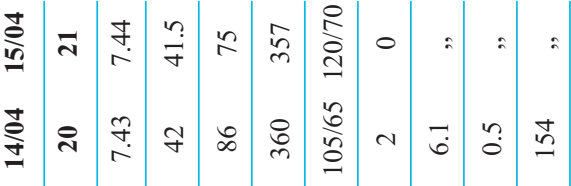

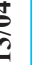

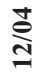

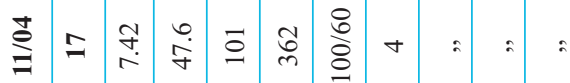

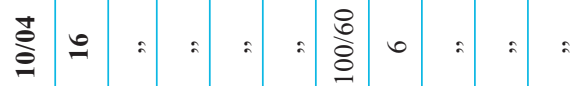

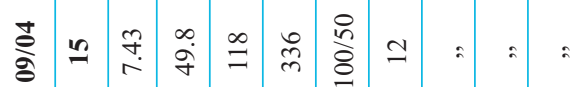

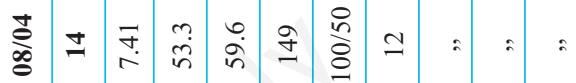

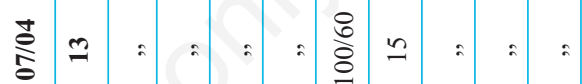

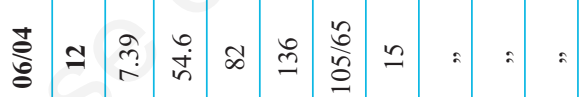

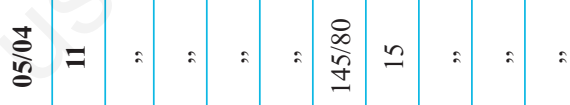

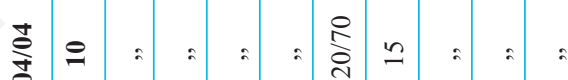

疍

$\stackrel{2}{8}$

政

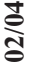

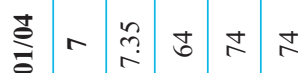

$\begin{array}{lll}8 & \\ 8 & n \\ 0 & n\end{array}$ (1) 
(Figure 2) revealed partial clearance of pulmonary infiltrates, while $\mathrm{pCO}_{2}$ decreased from $53 \mathrm{mmHg}$ to $49 \mathrm{mmHg}$, and $\mathrm{O}_{2}$ increased from $59 \mathrm{mmHg}$ to 118 $\mathrm{mmHg}$. The respiratory condition progressively improved, reaching spontaneous breathing on day 16 . Two consecutive nasal/throat RT-PCR swab samples were confirmed negative for RNA virus, so the patient was discharged on $29^{\text {th }}$ April. The comparison between respiratory parameters during CPAP and pharmacological therapy was presented in Table 2.

Just 2 months after discharge, the patient underwent a follow-up examination in our operative unit: she was fine, apyretic, alert, and eupneic. The physical examination revealed $\mathrm{SpO}_{2} 98 \%$ under ambient air and arterial blood pressure of $125 / 70 \mathrm{mmHg}$.

\section{Discussion}

Currently, there is no specific antiviral treatment for COVID-19. In the meantime, scientists are working hard to develop effective treatments. An antiviral drug must be able to target the specific part of a virus' life cycle that is necessary for it to reproduce. Viruses are highly adaptive. Because they reproduce so rapidly, they have the opportunity to mutate with each new generation, developing resistance to whatever drugs we develop.

Therapies under investigation include drugs used to treat malaria and autoimmune diseases, corticosteroids, and antibodies from people who have recovered from COVID-19. The association of azithromycin and hydroxychloroquine was the standard treatment, according to our local protocol. Azithromycin is an antibiotic used to prevent or treat co-infection by bacteria. Hydroxychloroquine proved to kill the COVID-19 virus in the laboratory dish. ${ }^{1}$ Hydroxychloroquine can predispose patients to lifethreatening arrhythmias. Hydroxychloroquine interferes with ventricular repolarization, leading to prolonged QTc interval and an increased risk of torsades de pointes. In our experience, it was challenging to correct hypokalemia because of the continuous renal potassium loss. The high prevalence of hypokalemia among patients with COVID-19 suggests the presence of disordered renin-angiotensin system activity, which increases due to reduced counteractivity of angiotensin-converting enzyme 2 , which is bound by COVID-19 pneumonia. ${ }^{2}$

Tocilizumab (TCZ), a humanized monoclonal antibody targeting the interleukin-6 receptor, seems a very promising drug. An important Italian study treated with TCZ 100 patients, the largest cohort until now, and found that the treatment was associated with a clinical improvement in more than three-quarters of patients. These preliminary results are encouraging, considering that the response to TCZ was rapid, within 12 to $72 \mathrm{~h}$, and sustained, as all patients with initial response continued to improve over the next ten days. ${ }^{3}$ TCZ efficacy needs to be validated in large clinical randomized trials.

In the context of great healthcare stress, Intensive

Table 2. Shows arterial blood gas during helmet-based continuous positive airway pressure (CPAP) and pharmacological therapy.

\begin{tabular}{|c|c|c|c|c|c|c|c|c|c|c|c|c|c|c|c|c|c|c|c|c|c|}
\hline Day of admission & 1 & 2 & 3 & 4 & 5 & 6 & 7 & 8 & 9 & 10 & 11 & 12 & 13 & 14 & 15 & 16 & 17 & 18 & 19 & 20 & 21 \\
\hline Hydroxychloroquine & $r$ & & & 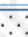 & $\therefore$ & 0 & 0 & 0 & 0 & 0 & 0 & 0 & 0 & 0 & 0 & 0 & 0 & 0 & 0 & 0 & 0 \\
\hline Azithromycin & $\therefore$ & $\because$ & & $\because$ & $\because$ & 0 & 0 & 0 & 0 & 0 & 0 & 0 & 0 & 0 & 0 & 0 & 0 & 0 & 0 & 0 & 0 \\
\hline Tocilizumab & 0 & 0 & $\beta$ & 0 & 0 & 0 & 0 & 0 & 0 & 0 & 0 & 0 & 0 & 0 & 0 & 0 & 0 & 0 & 0 & 0 & 0 \\
\hline Methylprednisolone & 0 & 0 & 0 & 0 & & & & & & & & 0 & 0 & 0 & 0 & 0 & 0 & 0 & 0 & 0 & 0 \\
\hline Morphine & 0 & 0 & 0 & 0 & & & & & & 0 & 0 & 0 & 0 & 0 & 0 & 0 & 0 & 0 & 0 & 0 & 0 \\
\hline Helmet CPAP & 0 & 0 & 0 & 0 & 8 & 28 & 88 & 88 & 0 & 0 & 0 & 0 & 0 & 0 & 0 & 0 & 0 & 0 & 0 & 0 & 0 \\
\hline
\end{tabular}

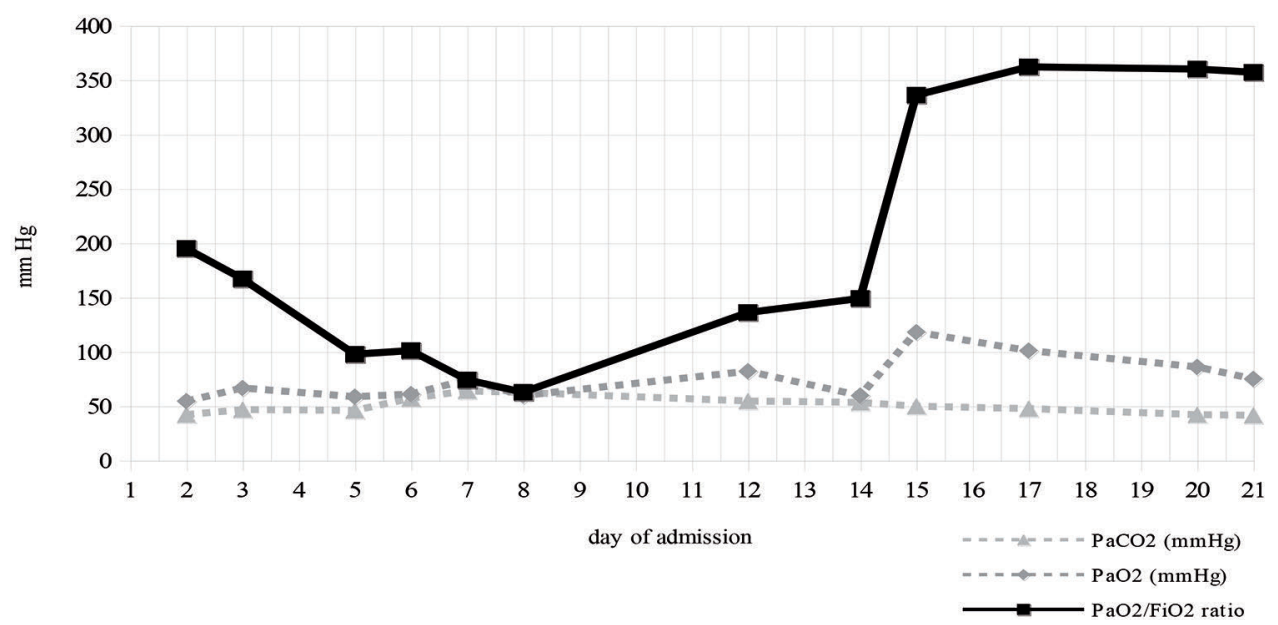

$\mathrm{PaCO}_{2}$, partial pressure of carbon dioxide; $\mathrm{PaO}_{2}$, partial pressure of oxygen; $\mathrm{FiO}_{2}$, fraction of inspired oxygen. 
Care Units may reach saturation. Thus, authorities converted internal medicine units into new respiratory intermediate care units, staffed by quickly trained personnel and equipped with noninvasive ventilation (NIV) devices. In our experience, we used helmet CPAP. Helmet CPAP was developed in 1991 in Italy, and is commonly used in emergency departments, in hospital and pre-hospital settings. In the COVID-19 pandemic, we have used helmet CPAP also in the internal medicine units to approach hypoxemic acute respiratory failure. Helmet CPAP confines aerosolized viral particle spread within the helmet. ${ }^{4}$ An independent metanalysis, including 4 randomized clinical trials that enrolled 377 patients, found that helmet CPAP significantly increased the $\mathrm{PaO}_{2} / \mathrm{FiO}_{2}$, decreased arterial $\mathrm{CO}_{2}$ levels, and reduced intubation rate and hospital mortality. ${ }^{5}$ In this case that we report, we stopped helmet CPAP for $\mathrm{CO}_{2}$ rebreathing. A possible problem related to helmet CPAP is the rebreathing of $\mathrm{CO}_{2}$. A traditional face mask is essentially an additional anatomical dead space, and its effect on $\mathrm{CO}_{2}$ rebreathing is proportional to its volume. Compared with the face mask, the helmet, due to its larger internal volume, which is always bigger than the tidal volume, might facilitate $\mathrm{CO}_{2}$ rebreathing. ${ }^{6}$

In this case, we used helmet CPAP to avoid severe complications and comorbidity after invasive mechanical ventilation through an endotracheal tube. Compared to invasive mechanical ventilation through an endotracheal tube, helmet CPAP is much safer and has fewer complications, such as upper airway trauma, laryngeal swelling, post-extubation vocal cord dysfunction, and nosocomial infection. Furthermore, in Down syndrome patients, many factors frequently subclinical or underdiagnosed may contribute to respiratory difficulties: hypotonia, tracheobronchomalacia, and adenoid hypertrophy, abnormal lung vasculature growth, alveolar hypoventilation, recurrent pulmonary infections, abnormal media of pulmonary arterioles, diminished alveoli density, gastroesophageal reflux. In DS patients, prolonged ventilation and hospitalization are required after invasive mechanical ventilation for cardiac surgery. ${ }^{7}$ A French single-center retrospective study evidenced the high mortality rate observed in children with DS admitted to the intensive care unit. The most frequent reason for admission was a respiratory disease. A history of mechanical ventilation was independently associated with death. This study has suggested that the advent of NIV could provide significant clinical improvement in children with DS who have upper airway obstruction, congestive cardiac failure, and pulmonary arterial hypertension. ${ }^{8}$

In the presence of dyspnea, we are used to giving low doses of morphine to avoid respiratory depression. Which role can play morphine in COVID19 pneumonia? Accompanying patients at the end of life remains a priority for healthcare teams everywhere. Every patient's right to receive analgesia and sedation until death to prevent all suffering must be guaranteed. Morphine may alleviate the symptoms of COVID-19: it lowers the respiratory rate and reduces shortness of breath. ${ }^{9}$ Thus, morphine causes a reduction of both the filling pressure and the arterial pressure, having a relaxing effect on the smooth muscles in both veins and arteries. Morphine reduces preload and afterload and has been frequently used in acute pulmonary edema. Moreover, in the hypothesis that COVID-19 is not a typical acute respiratory distress syndrome, because COVID-19 patients show significant vasoconstriction of small blood vessels, ${ }^{10}$ the vasodilator effect of morphine, should help the patient overcome the disease. Naturally, it is only a suggestion, and further validation with more substantial evidence is required.

\section{Conclusions}

COVID-19 became a public health emergency, causing tremendous stress on healthcare systems. In Northern Italy, internal medicine departments were reorganized entirely to face the emergency. This emergency well emphasizes the strategic role of Internal Medicine in the Italian Healthcare System. The admission of a patient with cognitive disability and chromosomic pathology may represent a huge challenge and request a person-centered and empathic approach. We used a CPAP helmet to avoid severe complications and comorbidity after invasive mechanical ventilation through an endotracheal tube.

\section{References}

1. Meo SA, Klonoff DC, Akram J. Efficacy of chloroquine and hydroxychloroquine in the treatment of COVID-19. Eur Rev Med Pharmacol Sci 2020;24:4539-47.

2. Chen D, Li X, Song Q, et al. Assessment of hypokalemia and clinical characteristics in patients with coronavirus disease 2019 in Wenzhou, China. JAMA Netw Open 2020;3(6) [Epub ahead of print].

3. Toniati P, Piva S, Cattalini M, et al. Tocilizumab for the treatment of severe COVID-19 pneumonia with hyperinflammatory syndrome and acute respiratory failure: A single center study of 100 patients in Brescia, Italy. Autoimmun Rev 2020;19(7) [Epub ahead of print].

4. Ing RJ, Corey B, Glenn M, et al. Role of helmetdelivered noninvasive pressure support ventilation in COVID-19 patients. J Cardiothorac Vasc Anesth 2020 May 8 [Epub ahead of print].

5. Liu Q, Gao Y, Chen R. Noninvasive ventilation with helmet versus control strategy in patients with acute 
respiratory failure: A systematic review and metaanalysis of controlled studies. Crit Care 2016;20:265.

6. Taccone P, Hess D, Caironi P, Bigatello LM. Continuous positive airway pressure delivered with a "helmet": effects on carbon dioxide rebreathing. Crit Care Med 2004;32:2090-6.

7. Joffe, Lesage F, Bustarret O, et al. Children with Down syndrome: clinical course and mortality-associated factors in a French medical pediatric intensive care unit. J Pediatr Child Health 2016;52:595-9.

8. Robert R, Le Gouge A, Kentish-Barnes N, et al.
Sedation practice and discomfort during withdrawal of mechanical ventilation in critically ill patients at end-oflife: a post-hoc analysis of a multicenter study. Intensive Care Med 2020;46:1194-203.

9. Pattinson KT. Opioids and the control of respiration. Clinical phenotypes of SARS-CoV-2: implications for clinicians and researchers. Br J Anaesth 2008;100:747-58.

10. Rello J, Storti E, Belliato M, Serrano R. Clinical phenotypes of SARS-CoV-2: implications for clinicians and researchers. Eur Respir J 2020;55(5) [Epub ahead of print]. 